\title{
THE INTEGRAL WEIGHT SYSTEM FOR TORUS ACTIONS ON SPHERES WITH NO FIXED POINT
}

\author{
ELDAR STRAUME
}

\section{Introduction.}

Let $G$ be a compact connected Lie group and $T$ a maximal torus. If $\varphi$ is a representation of $G$ on $\mathrm{R}^{n}$, then it is well known that $\varphi$ is determined by its restriction $\varphi \mid T$, the latter being completely described by its weight system, $\Omega(\varphi)=\Omega(\varphi \mid T)$. In the case of acyclic $G$-manifolds $X$, one obtains an analogous invariant, $\Omega(X)$, defined to be the weight system of the local representation of $T$ at a fixed point. It is called the geometric weight system of $(X, G)$, cf. Hsiang [4]. In a similar manner, if $X$ is a (cohomology) sphere and $T$ has fixed point, then one obtains such an invariant by local linearity, and it is a global invariant (i.e., independent of $x \in F(T)$; a result due to Atiyah and Bott when $F(T)=S^{0}$ ).

Now, assume $H^{*}(X ; \mathrm{Z})=H^{*}\left(S^{n} ; \mathrm{Z}\right)$ and $F(T)=\varnothing$. From Borel's formula, [1, p. 182], there is still a "connected" version $(p=0)$ as well as a $p$-version ( $p$ prime), $\Omega_{p}(X)$, of the geometric weight system. An alternative definition follows from the "splitting principle" in equivariant cohomology, see Hsiang [3]. In $\Omega_{0}(X)$ the weights are just directions in the weight lattice of $T$. In the past, efforts have been made to associate length to the weights in $\Omega_{0}(X)$, in a way which is consistent with local representations of subtori, see e.g. Sullivan [9]. Truly, the existence of such an integral weight system would be very pleasant, as it would simplify a lot of calculations of the orbit structural data, cf. Hsiang [3].

The cohomological method actually gives $\Omega_{0}(X)$ together with an "integral content" $C \in Z$. When $F(T) \neq \varnothing, C$ is the product of the coefficients of all nonzero weights, when they are expressed as multiples of primitive weights. In the opposite direction, however, one cannot always find a "good" integral weight system, as a refinement of $\Omega_{0}(X)$, by factorizing $C$ and distributing the factors as coefficients of weights. The reason is that the above mentioned consistency condition may fail. This is actually an interesting phenomenon which explains some of the exotic character of the action in question, see Sec. 4.

Received April 25, 1988; in revised form November 1, 1988. 
The purpose of this paper is to understand what is the "correct" notion of an integral weight system, $\Omega(X)$, and to establish some basic properties and its limitations. In Sec. 1 we review the definitions of $\Omega_{0}(X)$ and $\Omega_{p}(X)$, in Sec. 2-3 we discuss $\Omega(X)$. Applications of geometric weight systems, such as $\Omega_{0}(X), \Omega_{p}(X)$ (and to some extent $\Omega(X)$ ), can be found in the literature, we refer to [4], [5], [7], [8]. In a forthcoming paper, Hsiang, Straume [6], $\Omega(X)$ is needed to its full extent, and is playing the key rôle.

As an illustrative application of $\Omega(X)$ we shall classify all actions of $S^{1} \times S O(n)$, $n=2 k+1$, on spaces $X \sim_{z} S^{2 n-1}$ with $F\left(S^{1}\right)=\varnothing$. This will also cover a case left out by Wang [10] in his classification of actions of compact connected Lie groups on spheres with one-dimensional orbit space. The calculations are presented in Sec. 4.

\section{The rational weight system and the p-weight system.}

Let $X \sim_{0} S^{n}$ be a compact cohomology sphere and $T$ a torus acting on $X$. (We assume differentiability if necessary). The well known Borel formula (cf. [1]) gives

$$
\operatorname{dim} X-\operatorname{dim} X^{T}=\sum_{i=1}^{k}\left(\operatorname{dim} X^{H_{i}}-\operatorname{dim} X^{T}\right)=\sum m\left(H_{i}\right),
$$

where $H_{i} \subset T$ runs over all corank 1 subtori, and $m\left(H_{i}\right)$ may be called the multiplicity of $H_{i}$. The multiset of those $H_{i}$ contributing to the sum,

$$
\Omega_{0}^{\prime}(X)=\left\{H_{i} ; \text { mult. of } H_{i}=m\left(H_{i}\right)>0\right\},
$$

is called the (reduced) rational weight system of the $T$-space $X$. The "zero weight" (0) is given multiplicity $m_{0}=\operatorname{dim} X^{T}+1 \geqq 0$, and we write

$$
\Omega_{0}(X)=\Omega_{0}^{\prime}(X)+m_{0}\{0\} \text { (non-reduced) }
$$

Similarly, let $T_{p} \simeq\left(Z_{p}\right)^{r}$ be a $p$-torus, $p$ prime, acting on a space $X \sim_{p} S^{n}$. Then there is a Borel formula analogous to (1)

$$
\operatorname{dim} X-\operatorname{dim} X^{T_{p}}=\sum_{i}\left(\operatorname{dim} X^{K_{i}}-\operatorname{dim} X^{T_{p}}\right),
$$

where $K_{i} \subset T_{p}$ runs over subgroups $\simeq\left(Z_{p}\right)^{r-1}$. Those $K_{i}$ with multiplicity $m\left(K_{i}\right)=\left(\operatorname{dim} X^{K_{i}}-\operatorname{dim} X^{T_{p}}\right)>0$ are called the nonzero $p$-weights, and the total multiset of $p$-weights, $\Omega_{p}(X)$, is the p-weight system.

Let $T$ be a torus of rank $r$ and let $T_{p} \subset T$ denote its $p$-torus of rank $r$. Now, if $H \subset T$ is a subtorus of rank $r-1$, then $H \cap T_{p}=K$ is a $p$-torus of rank $r-1$. Hence there is a well defined restriction map

$$
\Omega_{0}^{\prime}(X) \rightarrow \Omega_{0}^{\prime}(X) \mid T_{p}, \quad H \rightarrow H \cap T_{p}=K
$$


between multisets. Here the multiplicity of $K$ is $m(K)=\sum m(H)$, sum over all $H$ such that $H \cap T_{p}=K$.

LeMma 1.1. $\Omega_{p}^{\prime}(X) \subset \Omega_{0}^{\prime}(X) \mid T_{p}$ (inclusion of multisets), if $X \sim_{p} S^{n}$.

PROOF. (i) Suppose $K \subset T_{p}$ is a corank $1 p$-torus which is not of type $H_{i} \cap T_{p}$, $H_{i} \in \Omega_{0}^{\prime}(X)$. We shall show $K \notin \Omega_{p}^{\prime}(X)$.

By assumption, $K H_{i}=T_{p} H_{i}, \forall H_{i} \in \Omega_{0}^{\prime}(X)$, and Borel's formula for the action of $T$ on $X^{K}$ gives

$$
\operatorname{dim} X^{K}-\operatorname{dim} X^{T}=\sum\left[\operatorname{dim}\left(X^{K}\right)^{H_{i}}-\operatorname{dim} X^{T}\right],
$$

where we can assume $H_{i} \in \Omega_{0}^{\prime}(X)$, since otherwise $X^{H_{i}}=X^{T}$ and hence $\left(X^{K}\right)^{H_{i}}=\left(X^{H_{i}}\right)^{K}=X^{T}$. But $\left(X^{K}\right)^{H_{i}}=X^{K H_{i}}=\left(X^{T_{p}}\right)^{H_{i}}$ and so

$$
\begin{aligned}
\operatorname{dim} X^{K}-\operatorname{dim} X^{T} & =\sum\left[\operatorname{dim}\left(X^{T_{p}}\right)^{H_{i}}-\operatorname{dim}\left(X^{T_{p}}\right)^{T}\right] \\
& \leqq\left[\operatorname{dim} X^{T_{p}}-\operatorname{dim} X^{T}\right]
\end{aligned}
$$

This implies $\operatorname{dim} X^{K}=\operatorname{dim} X^{T_{p}}$, so $X^{K}=X^{T_{p}}$ and $K$ is not a $p$-weight.

(ii) Let $K \in \Omega_{p}^{\prime}(\mathrm{X})$, and let $H_{i}, i=1,2, \ldots, q$, be those $H \in \Omega_{0}^{\prime}(X)$ satisfying $H_{i} \cap T_{p}=K$. We show $m(K) \leqq \sum_{i=1}^{q} m\left(H_{i}\right)$ :

Apply Borel's formula as follows. First, the $T$-action on $X^{K}$ gives

$$
\begin{gathered}
\operatorname{dim} X^{K}-\operatorname{dim} X^{T}=\sum_{i \leqq q}\left[\operatorname{dim} X^{H_{i}}-\operatorname{dim} X^{T}\right] \\
+\sum_{i>q}\left[\operatorname{dim}\left(X^{K}\right)^{H_{i}}-\operatorname{dim} X^{T}\right] \\
=\sum_{i \leqq q}\left[\operatorname{dim} X^{H_{i}}-\operatorname{dim} X^{T}\right]+\sum_{i>q}\left[\operatorname{dim}\left(X^{T_{p}}\right)^{H_{i}}-\operatorname{dim} X^{T}\right]
\end{gathered}
$$

Next, the $T$-action on $X^{T_{p}}$ gives

$$
\operatorname{dim} X^{T_{p}}-\operatorname{dim} X^{T}=\sum_{j}\left[\operatorname{dim}\left(X^{T_{p}}\right)^{H_{j}}-\operatorname{dim} X^{T}\right] .
$$

Combining the two formulas we find

$$
\begin{aligned}
m(K) & =\left(\operatorname{dim} X^{K}-\operatorname{dim} X^{T_{p}}\right)=\sum_{i \leqq q}\left(\operatorname{dim} X^{H_{i}}-\operatorname{dim} X^{H_{i} T_{p}}\right) \\
& \leqq \sum_{i \leqq q}\left[\operatorname{dim} X^{H_{i}}-\operatorname{dim} X^{T}\right]=\sum_{i \leqq q} m\left(H_{i}\right) .
\end{aligned}
$$


REMARK 1.2. If we had $X^{T_{p}}=\varnothing$ then $\Omega_{p}(X)=\Omega_{0}(X) \mid T_{p}$.

Let $T_{p}$ be a torus $(p=0)$ or $p$-torus ( $p$ prime). If $T_{p}^{\prime} \subset T_{p}$ is a $p$-subtorus (or torus, $p=0$ ), then we can calculate from $\Omega_{p}(X)$ its restriction to $T_{p}^{\prime}, \Omega_{p}(X) \mid T_{p}^{\prime}$ similar to (4). Another application of the Borel formula gives the following identity

$$
\Omega_{p}\left(X \mid T_{p}^{\prime}\right)=\Omega_{p}(X) \mid T_{p}^{\prime},
$$

where $\left(X \mid T_{p}^{\prime}\right)$ means $X$ regarded as $T_{p}^{\prime}$-space.

\section{On the definition of the integral weight system $\Omega^{\prime}(X)$.}

The weight lattice of the torus $T$ is

$$
\Gamma(T) \simeq H^{1}(T ; \mathrm{Z}) \simeq H^{2}\left(B_{T} ; \mathrm{Z}\right)=\mathrm{Z}\left[t_{1}, t_{2}, \ldots, t_{r}\right] .
$$

Let $\Omega$ be a multiset of weight pairs $\left( \pm \omega_{i}\right)$, each of multiplicity $m_{i}$, and possibly the zero weight of multiplicity $m_{0}$, say,

$$
\Omega=m_{1}\left\{ \pm \omega_{1}\right\}+m_{2}\left\{ \pm \omega_{2}\right\}+\ldots+m_{k}\left\{ \pm \omega_{k}\right\}+m_{0}\{0\} .
$$

The restriction of $\Omega, \Omega \mid T^{\prime}$, where $T^{\prime} \subset T$, is the "projection" of $\Omega$, as multiset, into the weight lattice $\Gamma\left(T^{\prime}\right)$. For example, the multiplicity of $\{0\}$ in $\Omega \mid T^{\prime}$ is $m_{0}+2 \sum m_{j}$, where $j$ runs over indices such that $\omega_{j} \mid T^{\prime}=0$.

We shall also regard the rational weight system $\Omega_{0}(X)$ of a $T$-space $X \sim S^{n}$ as an integral weight collection

$$
\Omega_{0}=m_{1}\left\{ \pm \bar{\omega}_{1}\right\}+m_{2}\left\{ \pm \bar{\omega}_{2}\right\}+\ldots+m_{k}\left\{ \pm \bar{\omega}_{k}\right\}+m_{0}\{0\},
$$

where $\left( \pm \bar{\omega}_{i}\right)$ is the unique pair of primitive weights such that $\bar{\omega}_{i}^{\perp}=H_{i}$, and $m_{i}=\frac{1}{2}\left(\operatorname{dim} X^{H_{i}}-\operatorname{dim} X^{T}\right)$ is its multiplicity.

We shall arrive at an appropriate definition of integral weight system by following the cohomological description of $\Omega_{0}^{\prime}(X)$ (or $\Omega_{p}^{\prime}(X)$ ). Recall the Borel construction of a fibration $X \rightarrow X_{T} \rightarrow B_{T}, X_{T}=E_{T} \times_{T} X$, and consider the Leray-Serre spectral sequence of

$$
\left(X, X^{T}\right) \rightarrow\left(X_{T}, B_{T} \times X^{T}\right) \rightarrow B_{T},
$$

where $X \sim_{0} S^{n}, X^{T} \sim_{0} S^{r}(r \geqq-1)$. Let $x \in H^{n}(X)=H^{n}\left(X, X^{T}\right), d f \in H^{r+1}\left(X, X^{T}\right)$ be generators (rational cohomology). The $E_{2}$-term $H^{*}\left(B_{T}\right) \otimes H^{*}\left(X, X^{T}\right)$ has two lines: 


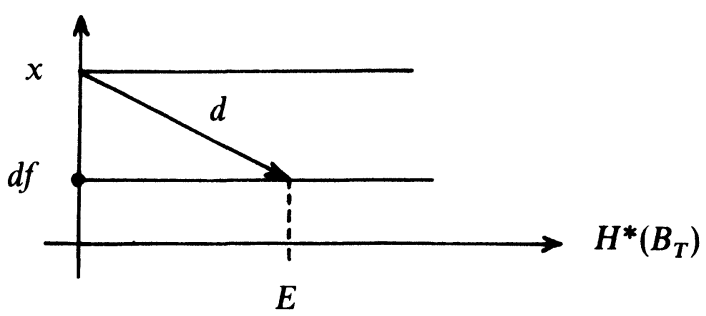

, the transgression of $x$ is

$$
d x=E_{T}(X) \otimes d f \in H^{n-r}\left(B_{T}\right) \otimes H^{r+1}\left(X, X^{T}\right) .
$$

By the well known splitting principle (cf. [3]) the so-called Euler class splits as follows

$$
E_{T}(X)=\prod_{i=1}^{k}\left(\bar{\omega}_{i}\right)^{m_{i}}(\bmod \text { constant factor })
$$

and so we recover $\Omega_{0}^{\prime}(X)=\sum m_{i}\left\{ \pm \bar{\omega}_{i}\right\}$.

Now, assume $X \sim_{Z} S^{n}$ and take cohomology with $Z$ as coefficients in the above spectral sequence. Then we obtain the (equivariant) Euler class

$$
E_{T}(X)= \pm C_{X} \cdot \prod_{i=1}^{k}\left(\bar{\omega}_{i}\right)^{m_{i}}, C_{X} \in \mathrm{Z}^{+},
$$

and we shall call $C_{X}$ the integral content. It contains information beyond $\Omega_{0}^{\prime}(X)$. In fact, we can do better, since $C_{X}$ has a canonical splitting into $k$ factors, as will be explained below.

Regard the spaces $X^{H_{i}}, H_{i}=\bar{\omega}_{i}^{\perp}$, as $T$-spaces, and observe that $\Omega_{0}^{\prime}\left(X^{H_{i}}\right)=m_{i}\left\{ \pm \bar{\omega}_{i}\right\}$, using Borel's formula. Therefore $E_{T}\left(X^{H_{i}}\right)= \pm C_{i}\left(\bar{\omega}_{i}\right)^{m_{i}}$ for some $C_{i} \in \mathrm{Z}^{+}$, and in Section 3 it will be seen that $C_{X}=\prod C_{i}$.

Definition 2.1. Assume $X \sim_{z} S^{n}$ is a $T$-space. The (reduced) integral weight system, denoted $\Omega^{\prime}(X)$, consists of its components, each component is an Euler class, namely

$$
\Omega^{\prime}(X)=\left\{E_{T}\left(X^{H_{i}}\right)\right\}_{i=1}^{k}=\left\{ \pm C_{i}\left(\bar{\omega}_{i}\right)^{m_{i}}\right\} .
$$

In particular, $X^{H_{i}}$ regarded as $T$-space, has only one (nonzero) component

$$
\Omega^{\prime}\left(X^{H_{i}}\right)=E_{T}\left(X^{H_{i}}\right)= \pm C_{i}\left(\bar{\varphi}_{i}\right)^{m_{i}} .
$$

$C_{i} \in \mathrm{Z}^{+}$is the integral content of the $i$-th component $\Omega^{\prime}\left(X^{H_{i}}\right)$ of $\Omega^{\prime}(X)$.

(In general, let $m_{0}=\operatorname{dim} X^{T}+1$. Then $\Omega(X)$ is $\Omega^{\prime}(X)$ plus a "zero-component" $m_{0}\{0\}$. This is the full (or unreduced) weight system. In particular, $\Omega^{\prime}(X)=\Omega(X)$ iff $X^{T}=\varnothing$.) 
REMARK 2.2. If $T$ acts linearly on $X=S^{n} \subset \mathrm{R}^{n+1}$ via the representation $\phi$ or if $T$ acts smoothly on $X \sim S^{n}$ with $X^{T} \neq \varnothing$ and $\phi=\phi_{x}$ is the local representation at $x \in X^{T}$, then

$$
E_{T}(X)= \pm \prod \omega_{i},
$$

where $\Omega^{\prime}(\phi)=\left\{ \pm \omega_{i}\right\}$ is the (reduced) weight system of $\phi$.

ReMARK 2.3. Let $X \sim_{z} S^{n}$ be a $G$-space, and $G$ is a compact Lie group with maximal torus $T$ and Weyl group $W$. Then $W$ acts on $H^{*}\left(B_{T}\right)$ and it is easily seen that $W$ permutes the classes $\pm E_{T}\left(X^{H_{i}}\right)$, that is, the integral weight system is $W$-invariant.

We now turn to the special case where $T$ has fixed point. In view of Remark 2.2 the following definition is consistent with Definition 2.1 , and is clearly a "refinement" of the latter.

Definition 2.4. Assume the action of $T$ on $X \sim S^{n}$ is differentiable, and $X^{T} \sim S^{d-1} \neq \emptyset$. Let $\phi_{x}$ be the local representation of $T$ at a fixed point $x$, and denote its weight system by $\Omega\left(\phi_{x}\right)$. Then the collection

$$
\Omega(X)=\Omega\left(\phi_{x}\right)+\{0\}=\Omega^{\prime}\left(\phi_{x}\right)+d\{0\}
$$

is defined to be the integral weight system.

Let $X$ be a $G$-space as above. Then $\Omega_{0}^{\prime}(X)=\sum_{j} m_{j}\left\{ \pm \bar{\omega}_{j}\right)$ splits into $W$-orbits

$$
\Omega_{0}^{\prime}(X)=\mu_{1} \Lambda_{1}+\mu_{2} \Lambda_{2}+\ldots+\mu_{q} \Lambda_{q},
$$

where the multiplicity $\mu_{l}$ is the common multiplicity $m_{j}$ of all $\left( \pm \bar{\omega}_{j}\right) \in \Lambda_{l}$. Suppose now $X^{T} \neq \varnothing$, and the action of $G$ is differentiable. Then the integral weight system $\Omega^{\prime}(X)=\Omega^{\prime}\left(\phi_{x}\right)$, where $\phi_{x}$ is the local representation of $T$ at some $x \in X^{T}$, is a "refinement" of $\Omega_{0}^{\prime}(X)$ which is clearly $W$-invariant (as multiset in the weight lattice $\Gamma(T))$, namely

$$
\Omega^{\prime}(X)=\delta_{1} \Sigma_{1}+\delta_{2} \Sigma_{2}+\ldots+\delta_{s} \Sigma_{s} \quad(s \geqq q),
$$

where $\Sigma_{i} \subset \Gamma(T)$ is a $W$-orbit of pairs $( \pm \omega)$.

If $T \subset G_{x}, G_{x}$ is an isotropy group, then the following identity holds

$$
\Omega^{\prime}(X \mid T)=\Omega^{\prime}\left(i_{x} \mid T\right)+\Omega^{\prime}\left(\mathscr{S}_{x} \mid T\right)=\Delta^{\prime}(G \mid T)-\Delta^{\prime}\left(G_{x} \mid T\right)+\Omega^{\prime}\left(\mathscr{S}_{x}\right)
$$

where $i_{x}$ is the isotropy representation of $G / G_{x^{\prime}} \Delta^{\prime}(G \mid T)=\Omega^{\prime}\left(\operatorname{Ad}_{G} \mid T\right)$ is the nonzero root system of $G$ (with respect to $T$ ) and $\mathscr{S}_{x}$ is the slice representation of $G_{x}$. There is an analogous formula for $p$-weights and $p$-roots, $p=0$ or prime. Application of formula (9) is demonstrated in and Sec. 4. 
Clearly, one obtains $\Omega_{0}^{\prime}(X)$ from $\Omega^{\prime}(X)$ by identifying weights which are colinear, and $\Omega^{\prime}(X)$ is a "refinement" of $\Omega_{0}^{\prime}(X)$, both in Definition 2.1 and 2.4. In the case of 2.1, where $F(T)$ is empty, one may wonder if it is possible to do something better, say, by somehow associate appropriate length to $m_{i}$ weight pairs parallel to $\left( \pm \bar{\omega}_{i}\right)$. Then the resulting multiset $\bar{\Omega}$ in the weight lattice could be regarded as the integral weight system $\Omega(X)$. Problems related to choosing such a collection, such as the validity of (8), (9), are postponed until $\S 5$.

\section{Further analysis of $\Omega(X)$ and equivariant Euler classes.}

Consider the family of $p$-groups $T_{p, \alpha} \subset T, \alpha \geqq 1$, where $T_{p, \alpha}=\left(Z_{p^{\alpha}}\right)^{r}, T=\left(S^{1}\right)^{r}$. The following result will be useful.

THEOREM 3.1. (Golber [2]). Let $X \sim_{\mathrm{Z}} S^{n}$ be a T-space (assume differentiability to avoid technical conditions). Let $C_{X} \in \mathrm{Z}^{+}$be the integral content of the Euler class $E_{T}(X)$, and for a fixed prime $p$ write $C_{X}=p^{e_{p}} \cdot C^{\prime}$, where $p \nmid C^{\prime}$. Then

$$
\sum_{\alpha \geqq 1}\left[\operatorname{dim} X^{T_{p, \alpha}}-\operatorname{dim} X^{T}\right]=2 e_{p} .
$$

THEOREM 3.2. In the above situation, let

$$
\Omega^{\prime}(X)=\left\{E_{T}\left(X^{H_{i}}\right)\right\}_{i=1}^{k}
$$

be the integral weight system (cf. Def. 2.1). Then

$$
\pm C_{X} \prod\left(\bar{\omega}_{i}\right)^{m_{i}}=E_{T}(X)=\prod_{i=1}^{k} E_{T}\left(X^{H_{i}}\right)
$$

namely, $C_{X}=\prod_{i=1}^{k} C_{i}$, where $C_{X}, C_{i}$ is the integral content of $E_{T}(X), E_{T}\left(X^{H_{i}}\right)$, respectively.

Proof. Write $C_{i}=p^{e_{p, i}} \cdot C_{i}^{\prime}$, where $p \nmid C_{i}^{\prime}$ is a fixed prime. Borel's formula for the $T$-action on $X^{T_{p, \alpha}}$ gives

$$
\begin{aligned}
\operatorname{dim} X^{T_{p, \alpha}}-\operatorname{dim} X^{T} & =\sum_{i}\left[\operatorname{dim}\left(X^{T_{p, \alpha}}\right)^{H_{i}}-\operatorname{dim} X^{T}\right] \\
& =\sum_{i}\left[\operatorname{dim}\left(X^{H_{i}}\right)^{T_{p, \alpha}}-\operatorname{dim} X^{T}\right]
\end{aligned}
$$

Golber's formula for the T-action on $X^{H_{i}}$ gives

$$
\sum_{\alpha}\left[\operatorname{dim}\left(X^{H_{i}}\right)^{T_{p, \alpha}}-\operatorname{dim} X^{T}\right]=2 e_{p, i}
$$


hence

$$
\sum_{i} \sum_{\alpha}\left[\operatorname{dim}\left(X^{H_{i}}\right)^{T_{p, \alpha}}-\operatorname{dim} X^{T}\right]=2 \sum e_{p, i}=\sum_{\alpha}\left[\operatorname{dim} X^{T_{p, \alpha}}-\operatorname{dim} X^{T}\right]
$$

Now, comparing with Golber's formula for the T-action on $X$, cf. (10), we get $e_{p}=\sum e_{p, \alpha}$. Consequently $C_{X}=\prod C_{i}$.

In order to study the behavior of the integral weight system with respect to restriction to subtori $T^{\prime} \subset T$, we shall first look more closely at (equivariant) Euler classes. Suppose $X^{T} \subset Y \underset{\ddagger}{\subset}$, where $Y \sim_{Z} S^{q}$ is $T$-invariant. In the spectral sequence of

$$
(X, Y) \rightarrow\left(X_{T}, Y_{T}\right) \rightarrow B_{T},
$$

the transgression of a generator $x \in H^{n}(X, Y ; Z)$ is $d x=E_{T}(X, Y) \otimes d y \in$ $H^{n-q}\left(B_{T}\right) \otimes H^{q+1}(X, Y)$. This gives the Euler class $E_{T}(X, Y) \in H^{n-q}\left(B_{T} ; Z\right)$; it is uniquely determined up to sign. By definition, $E_{T}\left(X, X^{T}\right)=\mathrm{E}_{T}(X)$.

Proposition 3.3. Let $X^{T} \subset Z \subset Y \subset X$ be $T$-invariant Z-cohomology spheres. Then

$$
E_{T}(X, Z)=E_{T}(X, Y) \cdot E_{T}(Y, Z) .
$$

Proof. Consider the three spectral sequences of the pairs

$$
\left(X_{T}, Y_{T}\right) \supset\left(X_{T}, Z_{T}\right) \supset\left(Y_{T}, Z_{T}\right)
$$

With the previous notation we may write

$$
\begin{aligned}
& d x^{\prime}=E_{T}(X, Y) \otimes d y^{\prime} \\
& d x=E_{T}(X, Z) \otimes d z \\
& d y=E_{T}(Y, Z) \otimes d z^{\prime}
\end{aligned}
$$

By the naturality of the above construction, induced mappings and (geometric) interpretation of transgression, we may identify $x$ and $x^{\prime}, y$ and $y^{\prime}, z$ and $z^{\prime}$, and then

$$
\begin{aligned}
d x^{\prime}=E_{T}(X, Y) \otimes d y^{\prime} & =E_{T}(X, Y) \cdot\left[E_{T}(Y, Z) \otimes d z^{\prime}\right] \\
& =E_{T}(X, Y) \cdot E_{T}(Y, Z) \otimes d z^{\prime} .
\end{aligned}
$$

Comparison with the second equation in (11) gives the product formula (essentially!).

Corollary 3.4. If $X^{T} \subset X^{T^{\prime}} \subset X, T^{\prime} \subset T$, then

$$
E_{T}(X)=E_{T}\left(X^{T^{\prime}}\right) \cdot E_{T}\left(X, X^{T^{\prime}}\right)
$$


Suppose $T^{\prime} \subset H_{j}$ for $j \leqq l$, and $T^{\prime} \notin H_{j}$ for $j>l$. By Theorem 3.2

$$
E_{T}\left(X^{T^{\prime}}\right)=\prod_{j \leqq l} E_{T}\left(X^{H_{j}}\right),
$$

so by Corollary 3.4

$$
E_{T}\left(X, X^{T^{\prime}}\right)=\prod_{j>l} E_{T}\left(X^{H_{j}}\right) .
$$

Let $i^{*}: H^{*}\left(B_{T}\right) \rightarrow H^{*}\left(B_{T^{\prime}}\right)$ be the map induced by the inclusion $T^{\prime} \stackrel{i}{\rightarrow} T$. It follows that

$$
E_{T^{\prime}}(X)=E_{T^{\prime}}\left(X, X^{T^{\prime}}\right)=i^{*}\left(E_{T}\left(X, X^{T^{\prime}}\right)\right)=\prod_{j>l} \pm C_{j}\left(i^{*} \bar{\omega}_{j}\right)^{m_{j}}
$$

Let $H_{j}^{\prime}, 1 \leqq j \leqq q$, be the different rational weights of the $T^{\prime}$-action on $X$; these are the corank 1 subtori of $T^{\prime}$ of type $\left(H_{t} \cap T^{\prime}\right)^{0}, t>l$. We also know $E_{T^{\prime}}(X)$ splits into the product of all $E_{T^{\prime}}\left(X^{H_{j}^{\prime}}\right)$, by Theorem 3.2.

Corollary 3.5. Let $T^{\prime} \stackrel{i}{\rightarrow} T, X \sim_{Z} S^{n}$ a $T$-space. Then the integral weight system of the $T^{\prime}$-space $X$

$$
\Omega^{\prime}\left(X \mid T^{\prime}\right)=\left\{E_{T^{\prime}}\left(X^{H_{j}^{\prime}}\right)\right\}_{j=1}^{q}
$$

can be calculated from $\Omega^{\prime}(X)=\left\{E_{T}\left(X^{H_{i}}\right)\right\}_{i=1}^{k}$ by

$$
E_{T^{\prime}}\left(X^{H_{j}^{\prime}}\right)=\prod_{t} i^{*}\left(E_{T}\left(X^{H_{t}}\right)= \pm \prod_{t} C_{t}\left(i * \bar{\omega}_{t}\right)^{m_{t}}\right.
$$

where $t$ runs over indices such that $\left(H_{t} \cap T^{\prime}\right)^{0}=H_{j}^{\prime}$, for each fixed $j$.

Corollary 3.6. Suppose all $m_{i}=1$, and hence we may write

$$
\Omega(X)=\left\{ \pm \omega_{1}, \pm \omega_{2}, \ldots, \pm \omega_{k}\right\}+m_{0}\{0\}, m_{0} \geqq 0 .
$$

If $\omega_{i} \mid T^{\prime}$ and $\omega_{j} \mid T^{\prime}$ are colinear when $i \neq j$, then by Corollary 2

$$
\Omega\left(X \mid T^{\prime}\right)=\Omega(X) \mid T^{\prime}
$$

\section{Exotic actions on spheres of cohomogeneity one.}

In this section we shall illustrate the usefulness of the integral weight system by studying some actions on spheres with 1-dimensional orbit space. The actions considered in Theorem 4.1 will also lead to more insight with regard to the integral weight system. We also refer to $\S 5$.

Consider the following orthogonal representation of $G=S^{1} \times S O(n)$, $n=2 k+1 \geqq 3$,

$$
\phi=\left[u_{1}^{2}\right]_{R} \oplus\left[\mu_{1}^{l} \otimes_{C} \rho_{n}^{C}\right]_{R}: C \oplus C^{n}=R^{2 n+2} \supset S^{2 n+1} .
$$


Let $( \pm \theta)$ be the unit weights for $S^{1}$, and $\Omega\left(\rho_{n}\right)=\left\{ \pm \tau_{i} ; i \leqq k\right\}+\{0\}$, so

$$
\Omega\left(S^{2 n+1}\right)=\Omega(\phi)=\{ \pm 2 \theta\}+\{ \pm l \theta\}+\left\{ \pm\left(l \theta \pm \tau_{i}\right)\right\} .
$$

Consider the Brieskorn variety $\Sigma^{2 n-1} \subset S^{2 n+1}$ defined by

$$
\Sigma^{2 n-1}: \begin{aligned}
& z_{0}^{l}+z_{1}^{2}+\ldots+z_{n}^{2}=0 \\
& \left|z_{0}\right|^{2}+\left|z_{1}\right|^{2}+\ldots+\left|z_{n}\right|^{2}=1 .
\end{aligned}
$$

It is clearly $G$-invariant, and as is well known, $\Sigma^{2 n-1} \sim_{z} S^{2 n-1}$ when $l$ is odd. (This does not hold if $l$ is even, see proof of 4.1.) It is easily seen that $\Omega_{0}\left(\Sigma^{2 n-1}\right)=\left\{ \pm\left(l \theta \pm \tau_{i}\right)\right\}+\{ \pm \theta\}$, and consequently

$$
\Omega\left(\Sigma^{2 n-1}\right)=\left\{ \pm q\left(l \theta \pm \tau_{i}\right)\right\}+( \pm d \theta\}, \text { cf. }(8) \text {. }
$$

By restriction to $S O(n)$ we first find $q=1$. Let $\Sigma^{1}=\left(\Sigma^{2 n-1}\right)^{H} \approx S^{1}$, $H=\theta^{\perp} \subset S O(n)$. Then $\Omega\left(\Sigma^{1}\right)=\{ \pm d \theta\}$ and

$$
\begin{aligned}
\Sigma^{1}: z_{1} & =z_{2}=\ldots=z_{n-1}=0 \\
z_{0}^{l}+z_{n}^{2} & =0, \quad \sum\left|z_{i}\right|^{2}=1 .
\end{aligned}
$$

Let $Z_{p} \subset S^{1}=T / H$, and observe that $\left(\Sigma^{1}\right)^{Z_{p}}=\emptyset$, so $p \nmid d$ and hence $d=1$,

$$
\Omega\left(\Sigma^{2 n-1}\right)=\{ \pm \theta\}+\left\{ \pm\left(l \theta \pm \tau_{i}\right)\right\} .
$$

Further analysis of this action will appear in Example 5.12.

In the remainder of this section we shall give a detailed proof of the following theorem.

THEOREM 4.1. Let $G=S^{1} \times \mathrm{SO}(n), n=2 k+1 \geqq 3$, and let $X \sim_{\mathrm{Z}} S^{2 n-1}$ be a faithful smooth G-manifold, and assume $X^{S^{1}}=\varnothing$. Then

(i) The integral weight system is

$$
\Omega(X)=\Omega\left(\Sigma^{2 n-1}\right), \text { as in (14), }
$$

$l$ is odd, and $X$ is homeomorphic to $S^{2 n-1}$.

(ii) $X$ can be imbedded G-equivariantly

$$
X \stackrel{\approx}{\rightrightarrows} \Sigma^{2 n-1} \subset S^{2 n+1} \subset \mathrm{R}^{2 n+2},
$$

where $\left(G, R^{2 n+2}\right)$ is the linear group described above, and $\Sigma^{2 n-1}$ is the Brieskorn variety, (12). [In particular, $X$ is the Kervaire sphere if $l \equiv \pm 3(\bmod 8)$ and $b P_{2 n} \simeq Z_{2}$ (i.e., the Kervaire invariant is trivial), and $X$ is the standard sphere otherwise.]

REMARK 4.2. (i) The theorem also gives that the Brieskorn variety $\Sigma^{2 n-1}$ is not an integral cohomology sphere when $l$ is even (well known, of course!).

(ii) The major part of the proof consists of establishing (15). Knowing $\Omega(X)$ we can determine precisely the isotropy types. Moreover, $\operatorname{dim} X / G=1(X / G$ is an 
interval), and the associated triple of isotropy groups, $\left(K_{1} \supset H \subset K_{2}\right)$, is uniquely determined modulo simultaneous conjugation in $G$. It follows that $X$ and $\Sigma^{2 n-1}$ are isomorphic as smooth $G$-manifolds.

First of all, since $F\left(S^{1}\right)=\varnothing$, every rational weight is of type $\pm(s \theta+\omega)$, where $\omega$ is a $\mathrm{SO}(n)$-weight, possibly $\omega=0$. By Weyl group invariance, and since $\# \Omega(X)=2 n$, it follows easily that

$$
\Omega(X)=\left\{ \pm q\left(l \theta \pm m \tau_{i}\right)\right\}+\{ \pm d \theta\}, \text { cf. (8) }
$$

for suitable positive integers, $q, l, m, d$.

$T=S^{1} \times T^{k} \subset S^{1} \times \operatorname{SO}(2 k) \subset G$ is the maximal torus in question, and

$$
\Omega(X)|\mathrm{SO}(n)=\Omega(X)| T^{k}=\left\{ \pm\left(q^{2} m^{2}\right)\left(\tau_{i}\right)^{2}\right\}_{i=1}^{k}+2\{0\},
$$

i.e., $\left(q^{2} m^{2}\right)=a b$ is the integral content of $\left\{ \pm a \tau_{i}, \pm b \tau_{i}\right\}$ in the local representation of $T^{k}\left(F\left(T^{k}\right) \simeq S^{1}\right)$. If $n \geqq 5$ then it is not too difficult to see, e.g., by (9), that $\Omega_{0}(X \mid \mathrm{SO}(n))=\Omega_{0}\left(2 \rho_{n}\right)$ implies $\Omega(X \mid \mathrm{SO}(n))=\Omega\left(2 \rho_{n}\right)$. If $n=3$ then the $\mathrm{SO}(3)$ action has weight system $\{ \pm a \tau, \pm b \tau\}+2\{0\}$, and we can show $a=b=1$ or $a=1, b=2$ (cf. [6]). In any case, $q=m=1$ in (16), so

$$
\Omega(X)=\left\{ \pm\left(l \theta \pm \tau_{i}\right)\right\}+\{ \pm d \theta\},(d, l)=1 .
$$

To see why $(d, l)=1$, observe that $\Omega_{p}(X)$ is calculable from $\Omega(X)$ (Lemma 5.4, Prop. 5.5), showing that $Z_{p} \subset S^{1}$ would act trivially if $p \mid(d, l)$, cf. also Prop. 5.8.

Let $T_{1}=\left(l \theta-\tau_{1}\right)^{\perp} \subset G_{x_{1}}=K_{1} . K_{1}^{0}$ is calculated precisely as in Example 5.11, using the corresponding formula (35). Now, the only multiple of $( \pm \theta)$ in $\Delta(G) \mid T_{1}$ is $\left( \pm \tau_{1}\right) \equiv( \pm l \theta)$, and $\Delta\left(K_{1}\right)$ cannot contain $\{ \pm l \theta)$ since $\mathrm{SO}(n) \notin K_{1}$. Consequently, $\{( \pm l \theta),( \pm 2 d \theta)\} \subset \Omega\left(X \mid T_{1}\right)$ and hence also by (34) (with $X$ instead of $\left.\Sigma^{2 n-1}\right)$

$$
\Omega\left(\mathscr{S}_{1}\right)=\{ \pm 2 d \theta\},\left(\mathscr{S}_{1}=\text { slice repr. at } x_{1}\right) .
$$

LEMma A. $K_{1}$ is connected.

Proof.

$$
\begin{aligned}
1 \rightarrow K_{1}^{0} \rightarrow K_{1} & \rightarrow E_{1} \rightarrow 0 \\
\bigcap_{N K_{1}^{0}} & =S^{1} \times \mathrm{SO}(2) \times \mathrm{SO}(n-2) .
\end{aligned}
$$

Suppose $Z_{p} \subset E_{1}$ with $p$ prime. Then $K_{1} \supset T_{p}=Z_{p} \times T_{p}^{k}$, where $\left(Z_{p}\right)^{k} \simeq T_{p}^{k} \subset T^{k}$. Therefore $X^{T_{p}} \simeq S^{1}$ and $p \mid d$ by (17). On the other hand, by (9), (17)

$$
X^{T_{p}} \subset X^{T_{p}^{k}}=X^{T^{k}}=X^{\mathrm{SO}(2 \mathrm{k})} .
$$

Consequently, $X^{K_{1}} \subset X^{\mathrm{SO}(2 k)}$ and $K_{1} \supset \mathrm{SO}(2 k)$, and this is a contradiction.

The slice representation of $K_{1}$ is $\left[\mu_{1}^{2 d}\right]_{R}$, cf. (18), and therefore the principal 
isotropy group is

$$
H=\left\{\left((\xi),\left(\begin{array}{cc}
\xi^{l} & 0 \\
0 & \mathrm{SO}(n-2)
\end{array}\right) ; \xi^{2 d}=1\right\} \simeq \mathrm{Z}_{2 d} \times \mathrm{SO}(n-2) .\right.
$$

We note that $Z_{2 d}$ sits "diagonally", namely, $\xi^{l} \neq 1$ if $\xi=\exp (2 \pi i / 2 d)$, except in the case $d=1$ and $l$ even, cf. (17).

Next, let $K_{2} \supset H$ be the other isotropy group of singular type; we can assume $\theta^{\perp}=T^{k} \subset K_{2}$, by the "Torus Algorithm" (cf. [5]), and (19) implies $K_{2}^{0}=\operatorname{SO}(2 k)$,

$$
\begin{aligned}
1 \rightarrow \mathrm{SO}(2 k) & \rightarrow K_{2} \rightarrow E_{2} \rightarrow 1 \\
& \operatorname{NSO}(2 k)=S^{1} \times S[0(2 k) \times 0(1)] .
\end{aligned}
$$

Now,

$$
H \subset K_{2} \Rightarrow\left[\exists h \in Z^{+}: 2 d \mid h, K_{2} \supset Z_{h} \times \mathrm{SO}(2 k) \subset S^{1} \times \mathrm{SO}(2 k)\right] .
$$

The slice representation $\mathscr{S}_{2}$ of $K_{2}$ has restriction $\mathscr{S}_{2} \mid \mathrm{SO}(2 k)=\rho_{2 k}$, and $\mathscr{S}_{2}$, having cohomogeneity one, is also irreducible

$$
\left\{\begin{array}{l}
\mathscr{S}_{2} \mid Z_{h} \times \operatorname{SO}(2 k)=\varphi_{1} \otimes_{\mathrm{R}} \rho_{2 k}, \operatorname{dim} \varphi_{1}=1 \\
1 \rightarrow Z_{h / 2} \rightarrow Z_{h} \stackrel{\varphi_{1}}{\longrightarrow} Z_{2} \rightarrow 1
\end{array}\right.
$$

Suppose $p \mid d$ with $p$ an odd prime. We see from $\mathscr{S}_{2^{\prime}}(22)$, that this does not give the "diagonal" group $Z_{2 d} \subset H$. Hence, the only possibility is $d=2^{s}(s \geqq 0)$.

LEMMA B. $d=1$.

Proof. Consider the 2-weight system $\Omega_{2}(X)$, with respect to the maximal 2-torus of $G$

$$
S=Z_{2} \times S^{\prime}=Z_{2} \times Z_{2}^{n-1}=\left\{\left(\varepsilon,\left(\begin{array}{ccc}
\varepsilon_{1} & & 0 \\
& \varepsilon_{2} & \\
& \ddots & \\
0 & & \varepsilon_{n}
\end{array}\right)\right) ; \varepsilon_{1} \varepsilon_{i}= \pm 1\right\} .
$$

Since $\Omega(X \mid \mathrm{SO}(n))=\Omega\left(2 \rho_{n}\right)$, we can show (see e.g. [7])

$$
\Omega_{2}(X \mid \mathrm{SO}(n))=\Omega_{2}\left(2 \rho_{n}\right)=2\left\{\varepsilon_{i} ; 1 \leqq i \leqq n\right\} .
$$

If we write

$$
\Omega_{2}(X)=a\{\varepsilon\}+b\left\{\varepsilon \varepsilon_{i}\right\}+c\left\{\varepsilon_{i}\right\}+\delta(0)
$$

then restriction to $\mathrm{SO}(n)$ implies $b+c=2, a+\delta=0$, so

$$
\Omega_{2}(X)=b\left\{\varepsilon \varepsilon_{i}\right\}+c\left\{\varepsilon_{i}\right\}, b+c=2 .
$$

Moreover, we can show $d>1 \Rightarrow b \geqq 1$ (by considering the "nonplitting" isotropy groups $H$ and $K_{1}$ ). From (24)

$$
X^{s}=X^{S^{\prime}}=\varnothing .
$$


Consider the 2-torus $S^{\prime \prime}=\mathrm{Z}_{2} \times T_{2}^{k} \subset S^{1} \times T^{k} \subset S^{1} \times \mathrm{SO}(2 k)$. It is given by $\varepsilon_{1}=\varepsilon_{2}, \varepsilon_{3}=\varepsilon_{4}, \ldots, \varepsilon_{2 k-1}=\varepsilon_{2 k}, \varepsilon_{n}=1$. We can calculate $\operatorname{dim} X^{S^{\prime \prime}}$ in two days:

From $\Omega(X): \operatorname{dim} X^{S^{\prime \prime}}=\left\{\begin{array}{r}1, d>1 \\ -1, d=1\end{array}\right.$

From $\Omega_{2}(X): \operatorname{dim} X^{S^{\prime \prime}}=\left\{\begin{array}{r}-1, b=2 \\ 0, b=1 \\ 1, b=0\end{array}\right.$

As observed above, $b=0$ is impossible if $d>1$, so the only possibility is $d=1$, $b=2$.

Suppose $l$ were even. Then $H \cap S^{\prime}=Z_{2}$ acts trivially. This is impossible, so $l$ must be odd.

Finally, it is easily seen that $E_{2}$ in (21) is a 2-group, and in fact $E_{2} \simeq Z_{2}$. Assuming $\mathrm{HC} \mathrm{K}_{2}$ we have more precisely

$$
K_{2}=\left\{\left(\varepsilon,\left(\begin{array}{cc}
\varepsilon & 0 \\
0 & O(n-1)
\end{array}\right)\right)\right\} \simeq S[O(1) \times O(n-1)] \simeq O(n-1) .
$$

This completes the proof of Theorem 4.1, cf. Remark 4.2.

REMARKS 4.3. (a) In the case of $n=7$, Theorem 4.1 also holds when $S O(7)$ is replaced by the subgroup $G_{2}$.

(b) The above actions are exotic (i.e., nonlinear) actions on homotopy spheres of cohomogeneity one. A complete discussion will appear in [6]. Wang [10] claims that such actions on spheres are of linear type, assuming $\operatorname{dim} X=N>31$ if $N$ is odd, or $N>4$ and is even. However, this is not true, and the above cases with $G=S^{1} \times S O(n)$ or $S^{1} \times G_{2}$ are missing in [10].

\section{Integral liftings and the consistency of $\Omega(X)$.}

Let $\Gamma$ be a multiset consisting of $k$ different pairs of primitive weights $\pm \bar{\omega}_{i}$ of multiplicity $m_{i}$, and suppose each $\pm \bar{\omega}_{i}$ is associated with some integer $C_{i}>0$. Then we write

$$
\Gamma=\left\{ \pm C_{1}\left(\bar{\omega}_{1}\right)^{m_{1}}+\ldots \pm C_{k}\left(\bar{\omega}_{k}\right)^{m_{k}}\right\} .
$$

$C_{i}$ is called the integral content along $\pm \bar{\omega}_{i}$, and $C=\Pi C_{i}$ is the total integral content. If we choose $m_{i}$ integers $c_{i j}$ with product $C_{i}$, and replace $\pm C_{i}\left(\bar{\omega}_{i}\right)^{m_{i}}$ in (25) by $\left\{ \pm c_{i 1} \bar{\omega}_{i}, \pm c_{i 2} \bar{\omega}_{i}, \ldots\right\}$, then we obtain a multiset $\bar{\Gamma}$ in the weight lattice, and $\bar{\Gamma}$ is called an integral lifting of $\Gamma$.

Suppose $T$ acts on $X$ with $X^{T}=\emptyset$, with $\Omega^{\prime}(X)=\Omega(X)$ defined as in Definition 2.1. Then $\Omega(X)$ is an object of type (25), with total integral content $C_{X}$. By definition, we also request that an integral lifting $\bar{\Omega}$ of $\Omega(X)$ must be $W$-invariant; in particular $\bar{\Omega}$ may be presented as in (8). In the special case where each $m_{i}=1$, $\bar{\Omega}$ is clearly unique and we may write $\bar{\Omega}=\Omega(X)$ without ambiguity. (In fact, we did so in \$4.) 
Now we turn to the following central problem.

Problem. Suppose $X \sim_{z} S^{n}$ is a $T$-space with $X^{T}=\emptyset$. Is it possible to obtain a suitable integral lifting $\bar{\Omega}$ of $\Omega(X)$ containing additional information about the orbit structure, similar to those of $\Omega^{\prime}(X)$ in the case $X^{T} \neq \emptyset$ ?

REMARK 5.1. It turns out that in general there is no unique "best possible" candidate $\bar{\Omega}(X)$ of integral lifting of $\Omega(X)$. In fact, even when it is unique it may still fail to fulfill certain consistency conditions which are known to hold if $X^{T} \neq \emptyset$, see below.

Let $\Omega(X)=\left\{ \pm \omega_{1}, \pm \omega_{2}, \ldots\right\}+m_{0}\{0\}$ be the weight system of an orthogonal action on $\mathrm{R}^{n+1} \supset S^{n}=X$. Then all representations $\phi \mid K, K \subset T$ can be calculated. In particular, $\forall K \subset T$

$$
\operatorname{dim} X^{K}=\#\left\{\left( \pm \omega_{j}\right) ; \omega_{j} \mid K=0\right\}+\left(m_{0}-1\right) .
$$

Clearly, if $X \sim S^{n}$ is a differentiable $T$-space and $X^{T} \neq \varnothing$, then the same formula (26) also holds (at least locally at $x \in X^{T}$ ).

Henceforth, assume $X \sim_{z} S^{n}, X^{T}=\varnothing$ (i.e., $m_{0}=0$ ). Among the various integral liftings $\bar{\Omega}$ of $\Omega(X)$ we hope to find a "good" candidate from which invariants like $\operatorname{dim} X^{K}$ can be calculated. So, we say $\operatorname{dim} X^{K}$ is calculable from $\bar{\Omega}$ if formula (26) holds. The question is whether such a $\bar{\Omega}$ actually exists.

Definition 5.2. Let $\bar{\Omega}$ be an integral lifting of $\Omega(X)$.

(i) $\bar{\Omega}$ is 0 -consistent if for each subtorus $T^{\prime} \subset T$ with $X^{T^{\prime}} \neq \varnothing, \Omega\left(X \mid T^{\prime}\right)=$ $\bar{\Omega} \mid T^{\prime}$.

(ii) $\bar{\Omega}$ is $p$-consistent if all numbers $\operatorname{dim}\left(X^{H_{i}}\right)^{T_{p, \alpha}}, \alpha \geqq 1, H_{i}=\bar{\omega}_{i}^{\perp}$ a rational weight, are calculable from $\bar{\Omega}$.

(iii) $\bar{\Omega}$ is consistent if all numbers $\operatorname{dim} X^{K}$ are calculable from $\bar{\Omega}$ whenever $K / K^{0}$ has prime power order, $K \subset T$ a closed subgroup.

Write $\bar{\Omega}=\bar{\Omega}(X)$, and for $T^{\prime} \subset T$ a subtorus, define

$$
\bar{\Omega}\left(X^{T^{\prime}}\right)=\left\{\left( \pm \omega_{i}\right) \in \bar{\Omega}(X) ; \omega_{i} \mid T^{\prime}=0\right\} .
$$

Clearly, $\bar{\Omega}\left(X^{T^{\prime}}\right)$ is an integral lifting of $\Omega\left(X^{T^{\prime}}\right)$.

LEMMA 5.3. (i) If $\bar{\Omega}=\bar{\Omega}(X)$ is 0-consistent, then $\bar{\Omega}\left(X^{T^{\prime}}\right)$ is also 0-consistent.

(ii) $\bar{\Omega}(X)$ is 0 -consistent if and only if $\Omega\left(X \mid H_{i}\right)=\bar{\Omega}(X) \mid H_{i}$ for each rational weight $H_{i}$.

Proof. (i) Let $T^{\prime \prime} \subset T$ be another torus and assume $\left(X^{T^{\prime}}\right)^{T^{\prime \prime}} \neq \varnothing$. Then $S=T^{\prime} \cdot T^{\prime \prime} \subset T$ is a torus and $X^{S} \neq \varnothing$. Hence, if $\bar{\Omega}$ is 0 -consistent, then $\Omega(X \mid S)=\bar{\Omega} \mid S$, and from the local representation of $S$

$$
\begin{aligned}
\Omega\left(X^{T^{\prime}} \mid T^{\prime \prime}\right) & =\Omega\left(X^{T^{\prime}} \mid S\right)\left|T^{\prime \prime}=\bar{\Omega}\left(X^{T^{\prime}}\right)\right| S \mid T^{\prime \prime} \\
& =\bar{\Omega}\left(X^{T^{\prime}}\right) \mid T^{\prime \prime} .
\end{aligned}
$$

(ii) This is clear since $X^{T^{\prime}} \neq \varnothing$ implies $T^{\prime} \subset H_{i}$ for some $i$. 
LEMMA 5.4. If $\bar{\Omega}=\bar{\Omega}(X)$ is 0 -consistent, then $\Omega_{p}(X)$ is calculable from $\bar{\Omega}$, namely

$$
\Omega_{p}(X)=\bar{\Omega}(X) \mid T_{p^{\prime}} \forall p\left(T_{p} \subset T\right) .
$$

In particular, $\operatorname{dim} X^{T} p$ is calculable from $\bar{\Omega}$.

Proof. By Lemma 1.1 each $p$-weight $K_{i} \subset T_{p}$ is a group of type $H_{j} \cap T_{p}$ for some rational weight $H_{j}$. Therefore $\operatorname{dim} X^{K_{i}}$ is calculable from $\bar{\Omega} \mid H_{j}=\Omega\left(X \mid H_{j}\right)$. Borel's formula gives

$$
\operatorname{dim} X-\operatorname{dim} X^{T_{p}}=\sum_{j}\left(\operatorname{dim} X^{H_{j} \cap T_{p}}-\operatorname{dim} X^{T_{p}}\right),
$$

and this gives $\operatorname{dim} X^{T_{p}}$ as well.

Now we turn to $p$-consistency ( $p$ fixed prime).

Lemma 5.5. Suppose $\bar{\Omega}=\bar{\Omega}(X)$ is p-consistent. Then $\Omega_{p}(X)$ is calculable from $\bar{\Omega}$, namely

$$
\Omega_{p}(X)=\bar{\Omega}(X) \mid T_{p} .
$$

Proof. There is a decomposition

$$
\bar{\Omega}(X)=\sum_{i=1}^{k} \bar{\Omega}\left(X^{H_{i}}\right)=\sum_{i=1}^{k} \sum_{\alpha \geqq 0} P_{i, \alpha},
$$

where $P_{i, \alpha}=\left\{( \pm \omega) \in \bar{\Omega}\left(X^{H_{i}}\right) ; \omega\right.$ is divisible by $p^{\alpha}$, but not by $\left.p^{\alpha+1}\right\}$, and we put $p_{i, \alpha}=\left(\frac{1}{2}\right) \cdot \# P_{i, \alpha}$ (number of pairs).

(i) We show $\operatorname{dim} X^{T_{p}}$ is calculable from $\bar{\Omega}$ : We claim

$$
\operatorname{dim} X^{T_{p}}+1=\sum_{i=1}^{k}\left[\operatorname{dim} X^{H_{i} \cdot T_{p}}+1\right]=2 \sum_{i=1}^{k} \sum_{\alpha>0} p_{i, \alpha} .
$$

The first equality follows from Borel's formula, the second from the fact that $\operatorname{dim} X^{H_{i} \cdot T_{p}}$ is calculable. The "outer" identity just means that $\operatorname{dim} X^{T_{p}}$ is calculable.

NOTE. In the same manner, each $\operatorname{dim} X^{T_{p, \alpha}}$ is calculable cf. section 3.

(ii) Let $K \subset T_{p}$ be a $p$-weight. By Lemma 1.1, $K=H_{i} \cap T_{p}$ for some $i$, and $K$ is a nonzero $p$-weight if and only if $\left(\operatorname{dim} X^{K}-\operatorname{dim} X^{T_{p}}\right)=m(K)>0$. We need to show that the numbers $m(K)$ are calculable.

Fix $K$, and we may re-index $\left\{H_{i}\right\}$ so that

$$
\begin{aligned}
& i \leqq q: K=H_{i} \cap T_{p}, \quad\left(X^{K}\right)^{H_{i}}=X^{H_{i}} \\
& i>q: K \neq H_{i} \cap T_{p}, \quad\left(X^{K}\right)^{H_{i}}=X^{H_{i} \cdot T_{p}} .
\end{aligned}
$$


Using Borel's formula for the $T$-action on $X^{K}$ :

$$
\begin{aligned}
\operatorname{dim} X^{K}+1 & =\sum_{i=1}^{q}\left(\operatorname{dim} X^{H_{i}}+1\right)+\sum_{i=q+1}^{k}\left(\operatorname{dim} X^{H_{i} \cdot T_{p}}+1\right) \\
& =2 \sum_{i=1}^{q} m_{i}+2 \sum_{i=q+1}^{k} \sum_{\alpha>0} p_{i, \alpha} .
\end{aligned}
$$

Now, $K \subset H_{i}$ for $i \leqq q$, and the double sum gives the number of pairs $( \pm \omega)$ such that $\bar{\omega}^{\perp} \neq H_{i}$ and $\omega \mid K=0$. Consequently, $\operatorname{dim} X^{K}$ is calculable.

Proposition 5.6. For each fixed prime $p, \Omega^{\prime}(X)$ has a p-consistent integral lifting $\bar{\Omega}$. Moreover, $\bar{\Omega}$ is unique modulo divisors of weights which are relatively prime to $p$.

Proof. Let $C_{i}=p^{e_{p, i}} \cdot C_{i}^{\prime}$ and $p \mid C_{i}^{\prime}$, where $C_{i}$ is the integral content of $\Omega^{\prime}\left(X^{H_{i}}\right)$. By Golber's formula (10)

$$
e_{p, i}=\sum_{\alpha \geqq 1} \sum_{\beta \geqq \alpha} p_{i, \beta}=\sum_{\alpha \geqq 1} \alpha \cdot p_{i, \alpha}
$$

Define $\bar{\Omega}\left(X^{H_{i}}\right)$ by taking $m_{i}$ copies of $\left( \pm \bar{\omega}_{i}\right)$ and let $p_{i, \beta}$ of them have coefficient $p^{\beta}$. This is possible since $m_{i} \geqq \sum_{\beta \geqq 1} p_{i, \beta}$. In order to have the integral content of $\bar{\Omega}\left(X^{H_{i}}\right)$ equal to $C_{i}$, we need only choose any factorization of $C_{i}^{\prime}$ and distribute the factors as additional factors of coefficients of any chosen weights.

Finally, put

$$
\bar{\Omega}=\sum_{i} \bar{\Omega}\left(X^{H_{i}}\right) .
$$

It is easily checked that $\operatorname{dim}\left(X^{H_{i}}\right)^{T_{p, \alpha}}$ is calculable from $\bar{\Omega}$. The uniqueness of $\bar{\Omega}$ (modulo non-p-primary factors) is also obvious.

Example 5.7. Let $X=\mathrm{SO}(3) / I \sim_{\mathrm{z}} S^{3}$ be the Poincare sphere with the transitive $\mathrm{SO}(3)$ action. The icosahedral group $I$ has order $60=4 \cdot 3 \cdot 5 . T=\mathrm{SO}(2)$ has no fixed point, whereas $Z^{Z_{2}} \simeq S^{1}, X^{Z_{3}} \simeq S^{1}, X^{Z_{5}} \simeq S^{1}, X^{Z_{p^{2}}}=\varnothing$ for $p$ prime. We infer from (10),

$$
\Omega(X)= \pm 30 \cdot\left(\gamma^{2}\right), \gamma=\text { unit weight of } T .
$$

REMARK 5.8. In general, one cannot tell from $\Omega^{\prime}(X)$ whether $T_{p, \alpha} \subset T$ acts trivially, or what is $\operatorname{dim} X^{T_{p, \alpha}}$. However, if each integral content $C_{i}$ of the components of $\Omega^{\prime}(X)$ satisfies $p^{2} \nmid C_{i}$, then $\operatorname{dim} X^{T_{p, \alpha}}$ is clearly calculable. So, in the above example, knowledge of the content $C=30$ gives all $\operatorname{dim} X^{Z_{p^{\alpha}}}$. It is also not generally possible to decide which of the groups $\left(T_{p} \cap H_{i}\right)$ are actually $p$-weights $\neq(0)$. The following proposition gives some partial results. 
Proposition 5.9. Let $\Omega^{\prime}\left(X^{H_{i}}\right)= \pm C_{i}\left(\bar{\omega}_{i}\right)^{m_{i}}$ be the components of $\Omega^{\prime}(X), i=1$, $2, \ldots, k$. (We assume $X^{T}=\varnothing$, so $\Omega^{\prime}(X)=\Omega(X)$.

(i) Suppose each $m_{i}=1$. If $p^{\alpha} \mid C_{i}, \forall i$, then $T_{p . \alpha}$ acts trivially on $X$.

(ii) If for some $i$, the highest power of $p$ dividing $C_{i}$ is $p^{q}$ and $q<m_{i}$, then $T_{p}$ does not act trivially on $X$. Furthermore, $K=T_{p} \cap H_{i}$ is a p-weight $\neq(0)$.

Proof. (i) Clearly $X^{H_{i}} \simeq S^{1}$, and from formula (10) we deduce $X^{H_{i} \cdot T_{p, \alpha}}=X^{H_{i}}$,

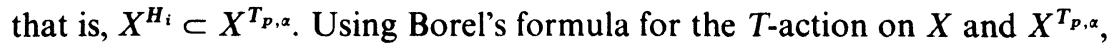

$$
\operatorname{dim} X+1=\sum\left(\operatorname{dim} X^{H_{1}}+1\right)=\operatorname{dim} X^{T_{p, \alpha}}+1,
$$

consequently $X^{T_{p, \alpha}}=X$.

(ii) If $T_{p}=T_{p, 1}$ acts trivially on $X^{H_{1}}$, then

$$
\begin{aligned}
2 m_{i} & =\left(\operatorname{dim} X^{H_{i}}+1\right) \leqq \sum_{\alpha \geqq 1}\left(\operatorname{dim} X^{H_{1} \cdot T_{p, \alpha}}+1\right) \\
& =2 e_{p, i}=2 q<2 m_{i},
\end{aligned}
$$

a contradiction. Hence, $T_{p}$ acts nontrivially on $X^{H_{i}}$.

It remains to show $m(K)=\left(\operatorname{dim} X^{K}-\operatorname{dim} X^{T_{p}}\right)>0$. But this number satisfies the relations in (5), and since there exists $i \leqq q$ such that $T_{p}$ acts nontrivially on $X^{H_{i}}$, we infer $m(K)>0$.

Let $G=G_{1} \times G_{2} \times \ldots \times G_{k}, k>1$, be a compact connected Lie group acting almost effectively on $X, T=T_{1} \times T_{2} \times \ldots \times T_{k} \subset G$ a maximal torus. A subgroup $K \subset G$ is splitting if $K=K_{1} \times K_{2} \times \ldots K_{k}, K_{i} \subset G_{i}$. In general, a closed subgroup $K$ is contained in a unique smallest splitting subgroup $\bar{K}$, called the splitting closure of $K$.

Observe that each $T_{i}$-weight may be regarded as a $T$-weight, in view of the projection $T \rightarrow T_{i}$ given by the above decomposition of $T$. We say the weight system $\Omega_{0}(X)$ is splitting if

$$
\left.\Omega_{0}^{\prime}(X)=\sum_{i=1}^{k} \Omega_{0}^{\prime}\left(X_{i}\right)=\sum_{i} \Omega_{0}^{\prime}\left(X \mid T_{i}\right)\right),
$$

where $X_{i}=X^{\grave{T}_{i}}, \grave{T}_{i}=T_{1} \times \ldots \hat{T}_{i} \times \ldots \times T_{k} . X_{i}$ can be regarded as a $T_{i}$-space. Hence each weight has "support" in one of the $T_{i}$. By Corollary 3.5 we also have

$$
\Omega^{\prime}(X)=\sum_{i} \Omega^{\prime}\left(X_{i}\right) \quad \text { (cf. Definition 2.1). }
$$

On the other hand, $X^{T_{i}} \neq \varnothing$ since $k>1$, and therefore $\Omega^{\prime}\left(X_{i}\right)$ has a well defined integral lifting given by the nonzero weights of the local representation; this lifting is also denoted $\Omega^{\prime}\left(X_{i}\right)$. Hence $\bar{\Omega}=\Omega^{\prime}(X)$ in (32) may be regarded as a "naturally" defined integral lifting of $\Omega^{\prime}(X)$, via representation theory, although $T$ itself has no fixed point. 
THEOREM 5.10. Assume $G=\prod G_{i}$ acts on $X \sim_{z} S^{n}$ with splitting weight system $\Omega(X), c f$. (32), and assume $\Omega(X)$ is consistent (cf. Definition 5.2, (iii)). Then all isotropy groups are splitting subgroups.

REMARK 5.11. The consistency assumption on $\Omega(X)$ in the above theorem is not really necessary; the complete proof is based upon Lemma 1.1, but we omit more details. Note that $\Omega(X)$ is consistent if $X^{T} \neq \emptyset$. The corresponding theorem for acyclic $G$-spaces $X$ is proved in Hsiang [4], and the proof below is similar to this since, at the technical level, "consistency" plays the role of having $X^{T} \neq \emptyset$.

PROOF. Suppose there is some nonsplitting isotropy group $G_{x}$. Then $\exists k \in G_{x}$ with $\pi_{j}(k) \notin G_{x}$, where $\pi_{j}: G \rightarrow G_{j}$. Let $K=\langle k\rangle$ be the closed subgroup generated by $k$, hence

$$
K=K^{0} \times Z_{m}, \quad \pi_{j}(K) \notin G_{x} .
$$

Let $K^{p} \subset \mathrm{Z}_{m}$ be the $p$-primary subgroup, and note that for some $p$ ( $p$ prime or zero) $K^{p} \notin G_{x}$, and denote this group $K^{p}$ by $Q$. We may assume $k \in T$, so $Q \subset \bar{Q} \subset T$, where $\bar{Q}$ is the splitting closure of $Q$.

Now, observe that $X^{\bar{Q}} \varsubsetneqq X^{Q}$ since $x \notin X^{\bar{Q}}$. On the other hand, $Q$ and $\bar{Q}$ are either tori or $p$-groups, so $\operatorname{dim} X^{\bar{Q}}<\operatorname{dim} X^{Q}$ by Smith theory. However, both numbers are calculable from $\Omega(X)$, and since the latter is splitting it is easy to see that the subgroup $Q$ and its splitting closure $\bar{Q}$ have $\operatorname{dim} X^{Q}=\operatorname{dim} X^{Q}$. This is a contradiction.

We conclude this section with a closer look at the concept of 0 -consistency.

QUESTION. Suppose all multiplicity coefficients $m_{i}=1$, so in particular $\Omega(X)$ has a unique integral lifting $\bar{\Omega}=\Omega(X)$. Is $\bar{\Omega} 0$-consistent?

Perhaps, the intuitive answer to the above question is "yes". However, as will be demonstrated below, the answer is generally "no".

To explain the situation, let $H_{i}$ be a (rational) weight in $\Omega_{0}(X)$, and let $\bar{\Omega}_{i}$ be the multiset of all nonzero weights in $\bar{\Omega} \mid H_{i}$. For a fixed weight direction (i.e. rational weight) $\pm \rho$ in $\bar{\Omega}_{i}$, let $m_{\rho}$ be the number of pairs in $\bar{\Omega}$ whose image in $\bar{\Omega}_{i}$ has the direction of $\pm \rho$, and let $C_{\rho}$ be the integral content of these image vectors. As a consequence of Corollary $3.5, \bar{\Omega}$ will be 0 -consistent if for each $H_{i}$ and choice of $\pm \rho$ one of the two conditions holds:

(i) $m_{\rho}=1$ (cf. Coroll. 3.6)

(ii) $C_{\rho}=1$ or a prime.

In contrast to the above, the following example has some $m_{\rho}=2$ and the corresponding $C_{\rho}=2 l, l$ odd. Explicit calculations show $\Omega(X)$ is not 0 -consistent. 
EXAMPLE 5.12. Consider again the linear group $\left(S^{1} \times S O(n), \phi\right)$ described in the beginning of $\S 4$, and its restriction to the Brieskorn variety $\Sigma^{2 n-1}$, see (12) and (14).

Let $T_{1}=\omega_{1}^{\perp} \subset T, \omega_{1}=\left(l \theta-\tau_{1}\right) . T_{1}$ is a maximal torus of some isotropy group $G_{x}$, since $\omega_{1} \notin \Delta^{\prime}(G)$ (= root system), according to the "Torus Algorithm", cf. [5]. From (14) we calculate weights $\neq 0 \bmod T_{1}$,

$$
\begin{aligned}
\Omega\left(\Sigma^{2 n-1}\right) \mid T_{1}: \pm \theta, & \pm\left(l \theta+\tau_{1}\right) \equiv \pm 2 l \theta \\
& \pm\left(l \theta \pm \tau_{i}\right) \equiv \pm\left(\tau_{1} \pm \tau_{i}\right), i>1
\end{aligned}
$$

Hence, by Corollary 3.5, $\Omega^{\prime}\left(\Sigma^{2 n-1} \mid T_{1}\right)$ coincides with the collection in (33), except that the subset $\{ \pm \theta, \pm 2 l \theta\}$ may be replaced by some $\{ \pm a \theta, \pm b \theta\}, a b=2 l$. However, by formula (9)

$$
\Omega^{\prime}\left(\Sigma^{2 n-1} \mid T_{1}\right)=\Delta^{\prime}\left(G \mid T_{1}\right)-\Delta^{\prime}\left(G_{x}\right)+\Omega^{\prime}\left(\mathscr{S}_{x}\right),
$$

with $\Delta^{\prime}(G)=\left\{ \pm\left(\tau_{i} \pm \tau_{j}\right)\right\}+\left\{ \pm \tau_{i}\right\}$, we find $\Delta^{\prime}\left(G_{x}\right) \supset\left\{ \pm\left(\tau_{i} \pm \tau_{j}\right) ; 1<i<j\right\}+$ $\left\{ \pm \tau_{i}, i>1\right\}$, and from this

$$
G_{x}^{0} \simeq\left\{\left(e^{i \theta},\left(\begin{array}{cc}
e^{i l \theta} & 0 \\
0 & * * * \\
* * *
\end{array}\right)\right)\right\} \simeq \mathrm{SO}(2) \times \mathrm{SO}(n-2)
$$

Now, $\Delta^{\prime}\left(G \mid T_{1}\right)-\Delta^{\prime}\left(G_{x}\right)$ contains $\pm \tau_{1} \equiv \pm l \theta$, so $a=2, b=l$, consequently

$$
\Omega\left(\Sigma^{2 n-1} \mid T_{1}\right) \neq \Omega\left(\Sigma^{2 n-1}\right) \mid T_{1} .
$$

\section{REFERENCES}

1. A. Borel et al., Seminar on Transformation Groups, Ann. of Math. Studies 46, Princeton 1961.

2. D. Golber, The cohomological description of a torus action, Pacific J. Math. 46, 1973.

3. W. Y. Hsiang, On the splitting principle and the geometric weight system of topological transformation groups I, Proceeding of the Second Conference on Compact Transformation Groups, Amherst, Mass. 1971, Lecture Notes in Maths. 298, 334-402, Berlin-Heidelberg-New York, Springer 1972.

4. W. Y. Hsiang, On the geometric weight system of differentiable transformation groups on acyclic manifolds, Invent. Math. 12 (1971), 35-47.

5. W. Y. Hsiang and E. Straume, On the orbit structures of SU(n)-actions on manifolds of the type of euclidean, spherical, or projective spaces, Math. Ann. 278 (1987), 71-97.

6. W. Y. Hsiang, E. Straume, Differentiable transformation groups on spheres with low cohomogeneities $I$, to appear.

7. E. Straume, p-weights and their application to regular actions of classical groups, Univ. of Oslo, 1975.

8. E. Straume, Weyl groups and the regularity properties of certain compact Lie group actions, Trans. A.M.S., Vol. 306, no. 1, 1988.

9. R. W. Sullivan, Linear models for compact groups acting on spheres, Topology 13 (1974), 77-87.

10. H. C. Wang, Compact transformation groups of $S^{n}$ with an $(n-1)$-dimensional orbit; Amer. J. Math. 82 (1960), 698-748. 